\title{
Erratum to: Multidimensional Feature Selection and Interaction Mining with Decision Tree Based Ensemble Methods
}

\author{
Lukasz Krol $^{(\bowtie)}$ and Joanna Polanska \\ Faculty of Automatic Control, Electronics and Computer Science, \\ Data Mining Group, Silesian University of Technology, Gliwice, Poland \\ \{lukasz.krol, joanna.polanska\}@polsl.pl
}

Erratum to:

Chapter "Multidimensional Feature Selection and Interaction Mining with Decision Tree Based Ensemble Methods" in:

F. Fdez-Riverola et al. (eds.), 11th International Conference on Practical Applications of Computational Biology \& Bioinformatics, Advances in Intelligent Systems and Computing 616, DOI 10.1007/978-3-319-60816-7_15

The original version of the book was inadvertently published with misspelt author name "Jonna Polanska" in Chapter 15 which should be corrected to read as "Joanna Polanska". The erratum chapter and the book have been updated with the change.

The updated online version of this chapter can be found at

http://dx.doi.org/10.1007/978-3-319-60816-7_15 